\title{
Aspectos cirúrgicos dos aneurismas isolados das artérias ilíacas
}

\author{
Surgical aspects of isolated iliac artery aneurysms \\ Aquiles Tadashi Ywata de C arvalho' ${ }^{1}$, Vanessa Prado', \\ H enrique Jorge $\mathrm{G}$ uedes N eto'2, Roberto Augusto $\mathrm{C}$ affaro ${ }^{3}$
}

\begin{abstract}
Resumo
O bjetivo: D iscorrer sobre os aspectos clínicos e o tratamento cirúrgico de uma série de casos de aneurismas isolad os das artérias ilíacas.

M étodos: Foram analisados retrospectivamente os dados protocolados e os prontuários de 12 pacientes com diagnóstico de aneurisma isolado das artérias ilíacas, operados no $D$ epartamento de Cirurgia da Santa Casa de São Paulo, no período de novembro de 1999 a fevereiro de 2003.

Resultados: A freqüência do aneurisma isolado das artérias ilíacas foi de $1,5 \%$ dos aneurismas abdominais operados no período do estudo. A faixa etária variou entre 56 e 80 anos, 33\% dos doentes apresentavam aneurisma bilateral, e os diâmetros dos aneurismas variaram entre 2,0 e $8,5 \mathrm{~cm}$. Em 83\% dos casos, os pacientes encontravam-se sintomáticos no momento do tratamento. Em nenhum dos casos 0 aneurisma se encontrava roto. A via de acesso utilizada nos aneurismas unilaterais foi a extraperitoneal homolateral à dilatação e, nos aneurismas bilaterais, a transperitoneal, longitudinal ou transversa. $\mathrm{N}$ ão dissecamos 0 segmento posterior das artérias ilíacas para clampeamento, para evitar a ocorrência de lesão venosa intra-operatória. $\mathrm{N}$ ão observamos mortalidade no período peroperatório. Em todos os casos, preservamos pelo menos uma artéria ilíaca interna.

Conclusão: A via de acesso para os aneurismas isolados das artérias ilíacas deve ser individualizada. A preservação de pelo menos uma artéria ilíaca interna constitui uma regra a ser observada, bem como deve-se evitar a dissecção circunferencial das artérias ilíacas no intraoperatório.
\end{abstract}

Palavras-chave: Aneurisma, artéria ilíaca, isolado, tratamento, cirurgia.

1. Pós-graduando, Disciplina de Cirurgia Vascular, Santa Casa de São Paulo, São Paulo, SP.

2. Professor assistente, Disciplina de Cirurgia Vascular, Santa Casa de São Paulo, São Paulo, SP.

3. Chefe, Disciplina de Cirurgia Vascular, Santa Casa de São Paulo, São Paulo, SP.

Artigo submetido em 09.11.05, aceito em 02.10.06.

J Vasc Bras 2006;5(3):203-8.

Copyright $\odot 2006$ by Sociedade Brasileira de Angiologia e de Cirurgia Vascular.

\begin{abstract}
O bjective: To discuss clinical aspects and surgical treatment of a series of cases of isolated iliac artery aneurysms.

Methods: Protocol data and medical charts of 12 patients with diagnosis of isolated iliac artery aneurysm were retrospectively analyzed. The patients underwent surgery at the D epartment of Surgery of Santa Casa de São Paulo, from N ovember 1999 to February 2003.

Results: Frequency of isolated iliac artery aneurysm was $1.5 \%$ of abdominal aneurysms who underwent surgery in the period under investigation. Age group ranged between $56-80$ years, $33 \%$ of patients presented bilateral aneurysm, and aneurysm diameters ranged between $2.0-8.5 \mathrm{~cm}$. In $83 \%$ of cases, the patients were symptomatic during treatment. Aneurysms were not ruptured in any case. Extraperitoneal approach ipsilateral to the dilatation was used in unilateral aneurysms, and transperitoneal approach, longitudinal or transverse, in bilateral aneurysms. Wedid not dissect the posterior segment of theiliac arteries for clamping, in order to avoid intraoperative venous lesion. There were no deaths during the surgery. In all cases, we preserved at least one internal iliac artery.

Conclusion: Approach to isolated iliac artery aneurysms should be chosen on an individual basis. Preserving at least one internal iliac artery is a ruleto be observed. Circumferential dissection of iliac arteries during the surgery should be avoided.
\end{abstract}

Key words: Aneurysm, iliac artery, isolated, treatment, surgery.

\section{Introdução}

0 aneurisma isolado das artérias ilíacas, sem comprometimento aórtico, representa uma afecção rara ${ }^{1-4}$, constando, na maioria das publicações, o relato de poucos casos.

Com prevalência significativamente maior no sexo masculino (5 masc::1 fem.) $)^{1,2}$, o aneurisma isolado da artéria ilíaca tem sua incidência variando de 0,03\% a 
$0,1 \% 1,3$ em adultos. Representa de 2 a $7 \%$ dosaneurismasintra-abdominais ${ }^{3,4}$, ocorrendo principalmentena sétima década de vida ${ }^{1,2}$.

A principal etiologia considerada, na maioria dos casos, tem sido o processo degenerativo da parede vascular $^{1,5}$. Em seguida, observam-se os aneurismas ateroscleróticos, infecciosos e micóticos ${ }^{1}$, além dos relacionados à gravidez, traumas, vasculites e doenças do colágeno ${ }^{2}$.

Apesar de geralmente permanecerem assintomáti$\cos ^{1,3,4}$, tendo seu diagnóstico durante exames rotineiros de imagem ${ }^{3}$, quando sintomáticos, os aneurismas das artérias ilíacas podem levar à dor, compressão de órgãos vizinhos, ruptura para estruturas adjacentes ${ }^{2}$, trombosee embolização ${ }^{4}$. São relatados sintomas vagos dedor abdominal eno quadril, queixas geniturináriase claudicação ${ }^{1}$. A tríade clássica de ruptura de um aneurisma abdominal com dor, hipoten são e massa abdominal pulsátil ${ }^{6}$ foi relatada em $38 \%$ dos pacientes com aneurisma de artéria ilíaca ${ }^{7}$, podendo atingir até $51 \%$ desses aneurismas ${ }^{5}$.

A abordagem cirúrgica eletiva é o tratamento de escolha2,3, sendo indicada para os doentessintomáticos e para aqueles com aneurismas da artéria ilíaca comum maioresque $2,5 \mathrm{~cm}^{8}$. D evido ao grandetamanho desses aneurismas à época do diagnóstico eà sua topografia na pelve, a cirurgia aberta representa um desafio, com riscos de hemorragia, secundária à lesão de veias adjacentes, elesões do trato urinário ${ }^{7}$. A tualmente, o tratamento endovascular desses aneurismas vem sendo descrito na literatura, mas ainda com poucos casos, devido à sua raridade, e resultados controversos $s^{3,4,9}$.

$\mathrm{N}$ a rotura, diversos trabal hos têm mostrado taxas de mortalidade elevadas nos pacientes submetidos a cirurgia de urgência, sendo os melhores resultados em torno de $33 \%$, podendo variar de 50 a $70 \%{ }^{2}$. M cC ready relatou que $58 \%$ dos indivíduos que se queixaram de dor abdominal já apresentavam rotura do aneurisma 8 . O s procedimentos terapêuticos eletivos, entretanto, apresentam menores taxas de mortalidade, variando de 0 a $11 \%$, de acordo com o serviço analisado ${ }^{2,9}$.

\section{0 bjetivos}

D escrever os dados clínicos, a técnica cirúrgica utilizada e os resultados pós-operatórios dos pacientes operados por aneurisma isolado das artérias ilíacas na D isciplina de C irurgia V ascular do D epartamento de Cirurgia da Santa Casa de São Paulo.

\section{Casuística e métodos}

Foram estudados, retrospectivamente, os prontuários e protocolos cirúrgicos de 12 pacientes consecutivos, portadores deaneurismaisolado dasartériasilíacas, operados no D epartamento de C irurgia da Santa C asa de São Paulo, no período de novembro de 1999 a fevereiro de 2003.

D escrevemos os dados clínicos dos pacientes, detaIhes da técnica cirúrgica empregada e a ocorrência de complicações intra e pós-operatórias nesta série de casos. Todos os casos foram operados pela mesma equipe cirúrgica, em um só hospital.

\section{Resultados}

A idade dos pacientes variou entre 56 e 80 anos, com média de 63,25 anos. T odos os pacientes realizaram exames de imagem no pré-operatório (Figura 1), sendo queo diâmetro dos aneurismas variou entre 2,0 e $8,5 \mathrm{~cm}$, com média de $4,3 \mathrm{~cm}$, segundo a tomografia pré-operatória. D o total dos nossos pacientes, $75 \%$ eram do sexo masculino.

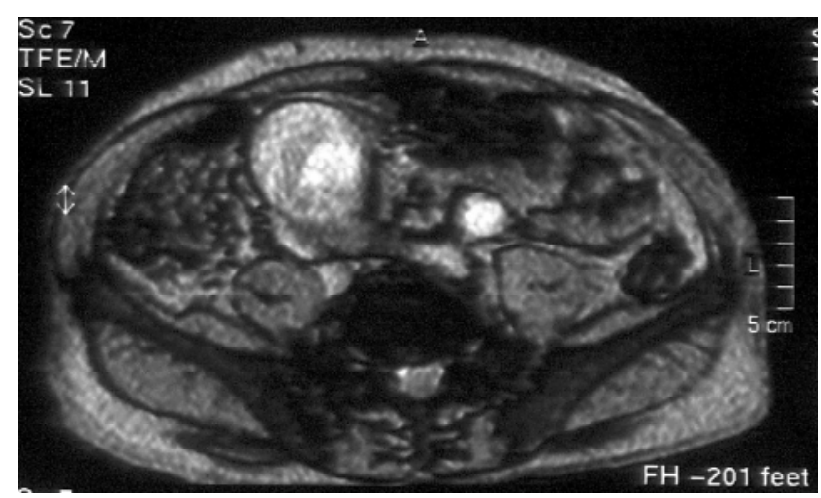

Figura 1 - Angiorressonância mostrando volumoso aneurisma de artéria ilíaca comum direita

Q uanto à presença de doenças associadas, 67\% dos pacientes eram hipertensos, 50\% eram tabagistas, $42 \%$ tinham doença pulmonar obstrutiva crônica, 33\% eram portadores de insuficiência cardíaca congestiva, e $25 \%$ eram diabéticos.

Q uanto à situação no retroperitôneo, 41,7\% dos aneurismas localizavam-seà direita, 33,3\% eram bilate- 
rais, e25\% localizavam-seà esquerda. Todosospacientes tinham aneurismas da artéria ilíaca comum, e dois destes casos apresentavam também aneurismas de uma das artérias ilíacas internas associados. Do total dos pacientes, $83 \%$ referiam dor abdominal ou pélvica de intensidade leve a moderada, sem sintomas do trato geniturinário ou retais.

o tratamento cirúrgico foi sempre realizado sob anestesia geral, e a via de acesso empregada se encontra descrita na Tabela 1. Após a exposição da dilatação aneurismática e dos colos proximal e distal do aneurisma, apenas na sua face anterior, era realizado o pinçamento arterial e abertura do saco aneurismático. 0 sangramento da artéria ilíaca interna era controlado sem o uso de pinças vasculares, através da insuflação do balão de uma sonda de Foley de tamanho apropriado (Figura 2). Esse tipo de abordagem operatória tem 0 objetivo de evitar a dissecção dessa artéria na pelve, diminuindo o risco delesões venosas inadvertidas, que, nessa região, podem ser de difícil correção e causa de sangramento volumoso.

$\mathrm{N}$ o caso de bilateralidade dos aneurismas, sem colo nas artérias ilíacas comuns, fez-se necessário o pinçamento da aorta distal, com confecção de derivação a partir da aorta. Os tipos de reconstruções arteriais empregadasseencontram pormenorizadosnaT abela 2 .

Preservamos pelo menos uma artéria ilíaca interna em todos os casos, $58 \%$ de preservação bilateral e $42 \%$ de preservação de apenasuma ilíaca interna. N os aneu- rismas unilaterais, quando a artéria ilíaca contralateral era pérvia, essa preocupação foi desnecessária. N os aneurismas bilaterais, a ilíaca interna foi, em geral, revascularizada diretamente através de prótese de politetrafluoroetileno (PTFE), com anastomose logo antes da bifurcação das ilíacas (Figura 3), ou, ainda, por perfusão retrógrada da anastomose distal na artéria ilíaca externa.

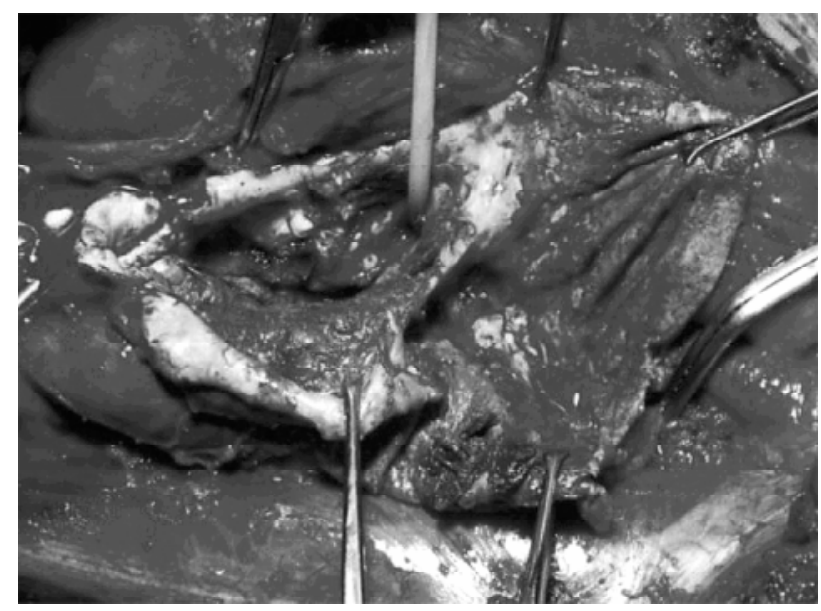

Figura 2 - Controle da artéria ilíaca interna através da introdução de sonda de Foley, evitando sua dissecção e o risco de lesão venosa iatrogênica

T abela 1 - V V ias de acesso empregadas conforme a situação do aneurisma

\begin{tabular}{lll}
\hline Situação & Via de acesso & N úmero de casos \\
\hline Aneurismas ilíacos unilaterais & Extraperitoneal direita & 5 \\
& Extraperitoneal esquerda & 3 \\
Aneurismas ilíacos bilaterais & Transversa infra-umbilical & 2 \\
& Extraperitoneal esquerda & 1 \\
& Laparotomia xifopúbica & 1 \\
\hline
\end{tabular}


T abela 2 - T Tipo de restauração vascular empregada após abertura do aneurisma

\section{Cirurgia realizada}

Enxerto em aorto-biilíaca externa

Enxerto em ilíaca comum/ilíaca externa

Enxerto em ilíaca comum/ilíaca comum ipsolateral

\section{N úmero de casos}

4

4

4

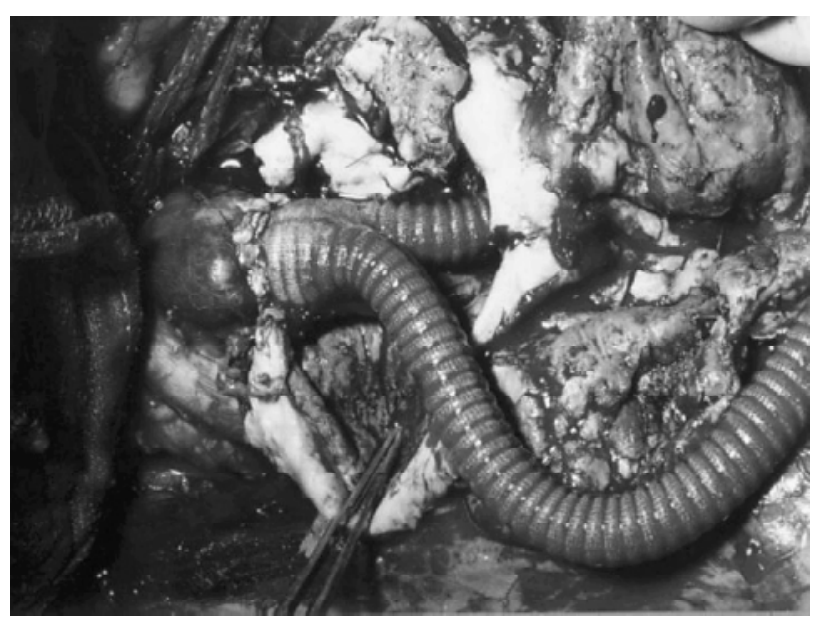

Figura 3 - Aspecto intra-operatório de revascularização aorto-bifemoral, em caso deaneurismabilateral de artériasilíacas comuns, com manutenção da perviedade da artéria ilíaca interna esquerda através deanastomoseno nível da bifurcação da artéria ilíaca comum esquerda

Q uanto às complicações intra-operatórias, não tivemos nenhum caso de lesão venosa iatrogênica, mas houve um caso de lesão ureteral unilateral, em um paciente com um grande aneurisma de artéria ilíaca comum, que englobava o ureter na sua parede. 0 ureter foi restaurado no intra-operatório, não trazendo outras complicações pós-operatórias ao paciente.

$\mathrm{N}$ o pós-operatório precoce, dois pacientes apresentaram uma pequena placa superficial, de aspecto isquêmico, na região sacral, sem repercussões clínicas e sem aspecto sugestivo de microembolização. Esses dois pa- cientes apresentavam lesões ateroscleróticas, associadas a doença aneurismática, nas artérias ilíacas internas. $\mathrm{N}$ enhum paciente evoluiu no pós-operatório com quadro clínico sugestivo de isquemia intestinal. U m paciente apresentou infecção superficial localizada da pele na região abdominal inferior. $\mathrm{N}$ ão houve mortalidade hospitalar na casuística descrita.

\section{D iscussão}

0 aneurisma isolado das artérias ilíacas corresponde de 0,9 a 2,2\% detodos os aneurismas intra-abdominais. D ois terços desses aneurismas acometem as artérias ilíacas internas, enquanto que $67 \%$ dos pacientes com aneurismas múltiplos envolvem a artéria ilíaca comum ${ }^{3,10}$. A incidência dessa afecção na $D$ isciplina de Cirurgia Vascular do D epartamento de Cirurgia da Santa C asa de São Paulo foi de 1,5\% dentre osaneurismas intra-abdominais operados no período do estudo.

Q uanto ao sexo, apenas $25 \%$ dos nossos pacientes eram do sexo feminino, semelhante ao trabalho de Krupski et al. ${ }^{9}$, que, em 21 pacientes operados de aneurisma de artérias ilíacas isolado, no período de 13 anos, teve apenas $19 \%$ dos pacientes do sexo feminino. A literatura confirma essa maior prevalência da doença no sexo masculino, com uma relação de cinco a 10 homens para uma mulher ${ }^{2,7}$.

A faixa etária encontrada em nossa casuística, com média de 63,5 anos, também está deacordo com outros autores. 0 s pacientes da série decasos deK rupski et al. ${ }^{9}$ tiveram uma média deidade de 69 anos, eN agarajan et al. relataram basicamente pacientes do sexo masculino e maiores de 60 anos $^{2,5}$. 
O utros autores publicaram os aneurismas das arté rias ilíacas variando entre 2,5 e $12 \mathrm{~cm}$ no seu diâmetro, no momento do seu reparo cirúrgico, ecerca de metade dos pacientes encontrava-se assintomática $5,10,11.0$ acometimento bilateral foi relatado em $48 \%$ dos casos, ea presença de dor abdominal em $19 \%$ dos pacientes 9 .

$\mathrm{N}$ anossa casuística, 33\% dos doentes apresentaram aneurismas bilaterais, com diâmetro variando entre 2,0 e $8,5 \mathrm{~cm}$ (média 4,3 cm); porém, diferimos da literatura em relação aos sintomas, pois $83 \%$ dos nossos casos encontravam-se sintomáticos no momento do tratamento, todos estes com queixa de dor abdominal inespecífica. $\mathrm{N}$ ão observamosem nenhum caso ossintomas urinários e neurológicos descritos na literatura ${ }^{9}$. T ambém não tivemos nenhum caso de erosão de estruturas adjacentes ou rotura.

A via de acesso utilizada nos aneurismas unilaterais foi a extraperitoneal homolateral, conforme utilizada pelamaioria dosautores. A via extraperitoneal esquerda também foi utilizada em um caso de aneurisma bilateral, com boa exposição da bifurcação da artéria ilíaca comum direita, mas, quando se deseja abordar a ilíaca externa contralateral mais distalmente, esse acesso pode oferecer dificuldades. A via transperitoneal é utilizada, na literatura, para o reparo dos casos de aneurisma de ilíaca bilateral ${ }^{9}$. Atualmente, temos preferido, nesses casos, a via de acesso transperitoneal transversa, que fornece acesso amplo às ilíacas comuns, internas e externas, bilateral mente, com baixa prevalênciadecomplicações pulmonares e da parede abdominal no pósoperatório.

0 tipo dereconstrução arterial dependeda extensão do comprometimento aneurismático das artériasilíacas e se há bilateralidade da doença. A correção cirúrgica através da interposição de enxertos sintéticos éa modalidadeterapêutica maisutilizada9,12. N a nossa casuísti$\mathrm{ca}$, todos os pacientes foram submetidos a cirurgia convencional através da interposição de enxerto sinté tico. Conforme orientado na literatura, optamos por preservar pelo menos uma artéria ilíaca interna em todos ospacientes, com o intuito deminimizar complicações como isquemia de cólon e da musculatura do quadrado lombar e glúteo.

$\mathrm{N}$ os aneurismas bilaterais da artéria ilíaca comum, a reconstrução arterial geralmente requer interposição deenxerto aorto-bifemoral. N essasituação, o reimplante da artéria mesentérica inferior pode ser necessário, principal mentesehouver necessidadedeexcluir as duas artérias ilíacas internas ${ }^{12}$. N a nossa casuística, reim- plantamos a artéria mesentérica inferior em dois casos, nos quais ela se encontrava calibrosa. $\mathrm{N}$ ão tivemos nenhum caso de isquemia de cólon diagnosticada no pós-operatório, mas, mesmo nos casos em que reimplantamos a artéria mesentérica inferior, houve a preservação de uma artéria ilíaca interna.

0 utra alternativa terapêutica é o tratamento endovascular, principalmente nos pacientes com alto risco cirúrgico ${ }^{2}$. A cirurgia endovascular apresenta algumas vantagens, como o acesso por punção percutânea da artéria femoral e menor morbidade ${ }^{2}$, sendo, por esse motivo, recomendada por alguns autores ${ }^{11}$, enquanto outros não tiveram bons resultados com essa técnica9 ${ }^{9}$.

A mortalidadehospitalar foi nula na nossa casuística. 0 utros trabalhos têm mostrado ausência de mortalidade na cirurgia do aneurisma isolado da artéria ilíaca ${ }^{9}$, recomendando, como fazemos aqui, o controle da ilíaca interna após abertura do saco aneurismático, evitando lesões venosas de difícil reparo. Também é importante não tentar dissecar circunferencialmente as artériasilíacas, pelo mesmo risco descrito acima. 0 bservando esse detal he, não tivemos nenhuma lesão venosa no intra-operatório.

\section{Conclusão}

A via de acesso para os aneurismas isolados das artérias ilíacas deve ser individualizada. A preservação de pelo menosuma artériailíaca interna constitui uma regra a ser observada, bem como deve-se evitar a dissecção circunferencial das artérias ilíacas no intra-operatório e da artéria ilíaca interna, o que diminui a ocorrência de lesões venosas de difícil correção.

\section{R eferências}

1. Richardson JW, Greenfield LJ. Natural history and management of iliac aneurysms. J V asc Surg. 1988;8:165-71.

2. N agarajan $M, C$ handrasekar $P$, Krishnan $E, M$ uralidharan $S$. Repair of iliac artery aneurysms by endoluminal grafting: the systematic approach of one institution. Tex H eart Inst J. 2000;27:250-2.

3. Brunkwall $J, H$ auksson $H$, Bengtsson $H$, Bergqvist $D$, Takolander R, Bergentz SE. Solitary aneurysms of the iliac arterial system: an estimate of their frequency of occurrence. J V asc Surg. 1989;10:381-4. 
4. Akiyama K, T akazawa A, H irota J, Yamanishi H, Akazawa T, $M$ aeda T . A double bifurcated graft for abdominal aorta and bilateral iliac artery reconstruction. Surg Today. 1999;29: 313-6.

5. Kanazawa $\mathrm{S}$, Inada $\mathrm{H}$, M urakami $\mathrm{T}$, Tabuchi $\mathrm{A}$, Ishida $\mathrm{A}$, T sunoda T. M anagement of isolated iliac artery aneurysms. J Cardiovasc Surg (T orino). 2000;41:513-4.

6. Levi N, Schroeder TV. I solated iliac artery aneurysms. Eur J V asc Endovasc Surg. 1998;16:342-4.

7. Lowry SF, K raft RO . I solated aneurysms of the iliac artery. Arch Surg. 1978;113:1289-93.

8. B rin BJ , Busuttil RW . I solated hypogastric artery aneurysms. Arch Surg. 1982;117:1329-33.

9. Krupski WC, Selzman CH , Floridia R, Strecker PK, N ehler M R, W hitehill TA. Contemporary management of isolated iliac aneurysms. J V asc Surg. 1998;28:1-11; discussion 11-3.

10. Best IM, Vansandani G, Bumpers H L. Complications of isolated bilateral iliac artery aneurysms. Am Surg. 2001;67: 767-71.
11. CentolaC, Anacleto A, A nacletoJC. T ratamento endovascular dos aneurismas das artérias ilíacas. In: Bonamigo T P, Riston $A V$, org. Aneurismas. Rio de Janeiro: Dilivros; 2000. p. 258-65.

12. T suji K, Saito M , M itani H . Surgical treatment of isolated iliac artery aneurysms. Jpn J V asc Surg. 2002;11:575-9.

Correspondência:

Aquiles T adashi Ywata de $C$ arval ho

Rua 0 ito de D ezembro, 291/601

CEP 40150-000 - Graça, Salvador

E-mail: aquiles tadashi@yahoo.com.br 